\title{
Phage engineering: how advances in molecular biology and synthetic biology are being utilized to enhance the therapeutic potential of bacteriophages
}

\author{
Russell Brown ${ }^{1,2}$, Andreas Lengeling ${ }^{3}$ and Baojun Wang ${ }^{1,2, *}$ \\ ${ }^{1}$ School of Biological Sciences, University of Edinburgh, Edinburgh, EH9 3FF, UK \\ 2 Centre for Synthetic and Systems Biology, University of Edinburgh, Edinburgh, EH9 3FF, UK \\ ${ }^{3}$ Infection and Immunity Division, The Roslin Institute and Royal (Dick) School of Veterinary Studies, University of Edinburgh, \\ Edinburgh EH25 9RG, UK \\ * Correspondence: baojun.wang@ed.ac.uk
}

Received October 8, 2016; Revised December 15, 2016; Accepted December 20, 2016

\begin{abstract}
Background: The therapeutic potential of bacteriophages has been debated since their first isolation and characterisation in the early $\mathbf{2 0}^{\text {th }}$ century. However, a lack of consistency in application and observed efficacy during their early use meant that upon the discovery of antibiotic compounds research in the field of phage therapy quickly slowed. The rise of antibiotic resistance in bacteria and improvements in our abilities to modify and manipulate DNA, especially in the context of small viral genomes, has led to a recent resurgence of interest in utilising phage as antimicrobial therapeutics.

Results: In this article a number of results from the literature that have aimed to address key issues regarding the utility and efficacy of phage as antimicrobial therapeutics utilising molecular biology and synthetic biology approaches will be introduced and discussed, giving a general view of the recent progress in the field.

Conclusions: Advances in molecular biology and synthetic biology have enabled rapid progress in the field of phage engineering, with this article highlighting a number of promising strategies developed to optimise phages for the treatment of bacterial disease. Whilst many of the same issues that have historically limited the use of phages as therapeutics still exist, these modifications, or combinations thereof, may form a basis upon which future advances can be built. A focus on rigorous in vivo testing and investment in clinical trials for promising candidate phages may be required for the field to truly mature, but there is renewed hope that the potential benefits of phage therapy may finally be realised.
\end{abstract}

Keywords: bacteriophage; phage therapy; phage engineering; synthetic biology

\section{INTRODUCTION}

The viruses of bacteria, or bacteriophages (phages), have a long and storied history [1] with even their initial identification becoming a matter of some debate. The

This article is dedicated to the Special Collection of Synthetic Biology, Aiming for Quantitative Control of Cellular Systems (Eds. Cheemeng Tan and Haiyan Liu). earliest known observation of what we would now consider to be a bacteriophage is ascribed to Frederick Twort [2], but the recognition of phages as a biological entity more in line with our current understanding is found in independent observations by Félix d'Herelle [3]. The contentiousness of phage spreads beyond their discovery, with questions regarding their efficacy and usefulness as treatment for bacterial disease persisting from their initial use in the 1920's to the modern day. There are two major reasons for this scepticism regarding 
the use of phage as therapeutic agents; firstly that their initial use was not always well regulated or documented in the English language and often highly regional [4], and secondly that in the antibiotic-era there has been little need for further investigation of a treatment method that is likely to be less reproducible, cost-effective, and efficacious than treatment of bacterial disease with antibiotic drugs. This lack of investment and testing means that many questions still remain open regarding the potential of phage therapy as a microbial control and treatment method, especially in the context of modern pharmaceuticals where methods of isolation and purification, target specificity, efficacy, pharmacokinetics, and safety are all major considerations. These are issues for which systematic regulated testing methodologies are well understood in the context of chemical antimicrobial drugs, but significantly less well defined in the context of replicative biological entities such as bacteriophage.

However, as we approach what may become a postantibiotic era of human and veterinary medicine $[5,6]$, the properties and therapeutic potential of phages has become an area of active interest. Co-incidentally this increased focus on phages has arrived at a point where the enabling effect of improved tools for genetic manipulation and characterisation are starting to be realised in the field of synthetic biology. Synthetic biology is enabling researchers to investigate new methods for controlling biological systems [7-9] and how best to harness these concepts in the context of applied research and medicine [10-12]. The relatively small size of the genomes of phages, and the relative ease of propagation and manipulation within a laboratory environment when compared to eukaryotic viruses, has meant that bacteriophages are ideal candidates for investigation of genetic modification of viruses $[13,14]$, with a specific focus on whether genetic modification can be useful in the context of generating phage suitable for treatment of human and veterinary bacterial diseases $[15,16]$.

Genetic modification of phages is specifically being applied to address some of the concerns regarding aspects of their biology that may be important in the context of treatment of disease. These include modification of the host-range of phages in order to enable targeted treatment of bacterial populations, methods to increase the persistence of phages within the host and modulation of phage interactions with the host immune system, and altering the life-cycle of a phage to minimise the risk of host-toxicity effects upon lysis of a bacterial population in vivo. This review will discuss specific results associated with the genetic modification of phages; specifically in the context of the importance of phages as engineered novel antimicrobial therapeutic agents.

\section{ENGINEERING BACTERIOPHAGE WITH AN EXPANDED OR ALTERED HOST-RANGE}

One of the major challenges of harnessing bacteriophage to treat bacterial infection is the huge diversity of both bacteria and phages. Viral genomes are typically highly plastic, and possibly due to the interaction of phages with bacterial species that also show a high degree of genetic plasticity and adaptability, phages appear to have evolved in such a way as to become highly specified infectious agents. When considering the clinical use of phages this tightly defined host-range becomes extremely important. Precise targeting is a prized goal for any treatment methodology, but when a number of different species and intra-species variants need to be treated, as would be the case in the treatment of a typical bacterial infection, there needs to be flexibility in the system to target a significant proportion of these variants at the infection site. Therefore investigation into ways to broaden or alter the host-range of phages is an important step towards their relevance in a clinical setting.

Attachment and delivery of viral genome to the host is mediated by the tail fibers and baseplate of phage, with tail fibers often specific to a certain bacterial outer membrane component, and locationally sensitive due to differing densities of these membrane components at different sites on the surface of the host. Therefore, these mediators of attachment and adsorption are ideal targets when considering methods to alter host-range (Figure 1). A discussion of the full range of attachment sites available to bacteriophage is beyond the scope of this article, with a vast array of host surface components such as flagella, pili, capsules, lipopolysaccharides and proteins all being implicated as sites for phage attachment, but a detailed summary can be found in Rakhuba et al. [17]. The binding of tail fibers to the cell surface typically takes place as either an abortive on-off interaction or as an irreversible/strong interaction, and in many cases it is only when a certain proportion of tail fibers have been adsorbed to the cell surface in an irreversible manner that DNA penetration proceeds [18-24]. The baseplate also plays a role in attachment and entry, especially in phages with contractile tail sheaths such as T4-like phages, where initial binding of long tail fibers leads to a confirmation change in the baseplate, which both presents short tail fibers to mediate irreversible binding and simultaneously contracts the tail sheath to expose the inner hollow tube and puncture the host cell membrane ready for genome delivery [25-27].

In the context of bacteriophage therapies, broadening of host-range was initially achieved by utilising a mixed population of phages in a single treatment, typically 


\section{Mixed wild-type phage population}
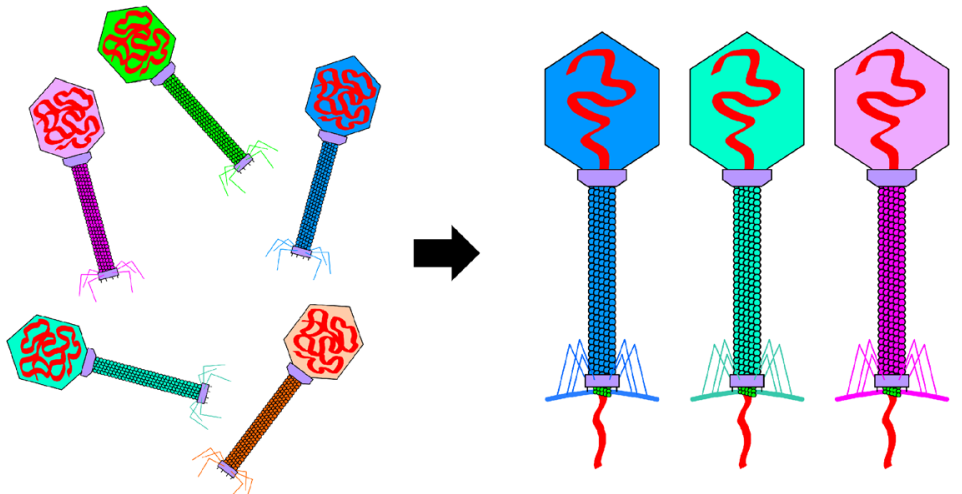

Mixture of wild-type phage with varied host-ranges

Each wild-type phage is able to infect a specific host within a mixed bacterial population

\section{Swapping of tail fibers}

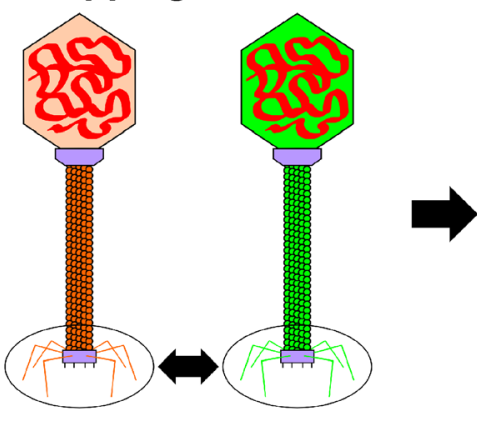

Tail fiber genes swapped between 2 wild-type phage

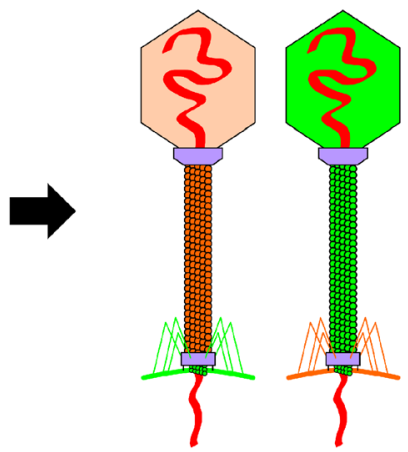

Host-range altered dependant upon tail fiber origin

\section{Generation of tail fiber diversity}

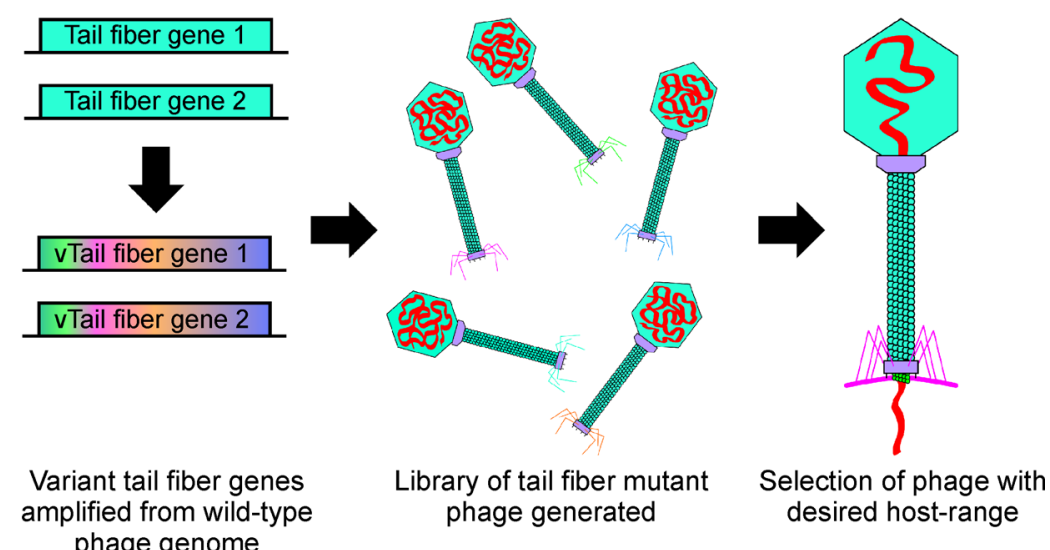

Figure 1. Schematics showing three representative methods used to generate or engineer a population of phages with altered or broadened host-range. The traditional approach to expanding treatment specificity involves isolating a number of wild-type phages with broadly similar lytic properties but with different host-specificities. When these phage are mixed and applied to a bacterial population the diversity of available host-ranges allows for the concurrent targeting of a number of different bacterial species. A second approach involves using genome engineering to swap tail fiber genes between two phage. Generally this approach will involve swapping tail fibers from a broadly infectious but poorly lytic (or lysogenic) phage into the genome of a highly lytic phage. By doing this, it is hoped that broad-infectivity is transferred to the engineered phage through exchanged tail fibers whilst retaining the lytic properties of the wild-type phage, thus expanding the host-range. The third approach involves randomly altering the tail fiber genes of a phage to generate a library of variants. Here, polymerase chain reaction (PCR) mutagenesis is used to amplify a pool of tail fiber variants from the wild-type genome. This gene library can then be subsequently transferred into the wild-type phage genome by homologous recombination. It is then possible to rescue a library of phages with different tail fiber mutations that can then be screened for the ability to infect a mixed population of bacteria, or for the ability to infect a specific host that could not be infected by the wild-type phage. 
called a phage cocktail [28-31] (Figure 1). This combination method appears to be tested with a high degree of efficacy, especially in Eastern Europe [4], but it is perhaps difficult to envisage the transition of such a treatment strategy into a modern Western regulatory and manufacturing framework, where the precise quantities and qualities of each phage would likely need to be characterised and quantified pre- and post-mixing. However, defined methods for phage cocktail design, isolation and preparation have been developed and this is still very much a viable strategy for future implementations of phage therapy [32-36]. However, phage engineering has aimed to reduce the necessity for delivery of a diverse population of phage in a cocktail by directly modifying the mediators of attachment in a lytic phage 'scaffold' which can then be used to produce a population of phages capable of lysing bacteria not typically targeted by the wild-type 'scaffold'. Compatibility and conserved genome organisation between phages allows for the direct swapping of tail fiber genes between phages from different genera and, due to host-range being closely correlated with tail fiber origin, this has allowed the direct manipulation of phage host-range (Figure 1). For example Mahichi et al. demonstrated that transfer of the tail fiber genes gp 37 and gp 38 from the broadly infectious phage IP088 to the lytic but narrow-spectrum phage T2 led to an expansion of the T2 host-range without loss of lytic function [37]. Similarly, although not an example of hostrange expansion, Yoichi et al. demonstrated that the ability of phage PP01 to recognise and bind the highlypathogenic E.coli O157:H7 could be transferred by recombination of the $g p 37$ and gp38 genes into T2 [38]. However, whilst the modified T2 was now able to infect O157:H7, it presented a smaller plaque phenotype and lower adsorption efficiency with an increase in the number of phage-resistant bacteria compared to wildtype PP01. A different strategy has been employed to modify the host-range of T4, here Pouillot et al. generated a library of virulent T4 phage capable of infecting a broad spectrum of bacterial species by utilising random PCR mutagenesis of $g p 37$ and $g p 38$ followed by homologous recombination to generate $\mathrm{T} 4$ tail fiber mutants [39]. However, it should be noted that this paper does not exhaustively address the question of whether the lytic properties of the phage are comparable between wild-type T4 in an E. coli host and the modified T4 library in Pseudomonas aeruginosa and Yersinia ruckeri. Phages T7 and T3 have also been targets for investigation due to their genome homology and the relatively large historical body of work regarding their characterisation [40]. A study by Lin et al. into differences in F-plasmid mediated genome entry exclusion in phages $\mathrm{T} 7$ and $\mathrm{T} 3$ when infecting male $\left(\mathrm{F}^{+}, \mathrm{F}^{\prime}, \mathrm{Hfr}\right)$ and female $\left(\mathrm{F}^{-}\right)$strains of $E$. coli identified that recombination between the two phages led to a T3/7 hybrid containing a T7-like gp 17 tail fiber gene [41]. The T3/7 phage showed altered adsorption efficiencies in four strains of $E$. coli when compared to wild-type T3, which indicated that the host-range had become more T7-like upon integration of the T7-like gp17 tail fiber. However, when the plating efficiencies (EOP) of wild-type T7 and T3 phages and the T3/7 phage were compared in the male K91 strain of E. coli, the EOP of T3/7 was 100-fold higher than T7 and 10,000-fold higher than T3 [41]. This result shows that whilst exchange of tail fibers may be a key starting point in host-range modification, altered adsorption characteristics may lead to unforeseen interactions with other properties of the phage. In this case, once adsorption efficiencies had been improved on the male strain, the presence or activation of genes in the hybrid $\mathrm{T} 3 / 7$ phage mediated escape from $\mathrm{F}$ exclusion with an efficiency not observed in either wild-type parental phage, and this property acted as a secondary mechanism to broaden the phage hostrange. The basic principles of creating $\mathrm{T} 7$ and $\mathrm{T} 3$ hybrids was greatly expanded upon in the work of Ando et al. where variants of both $\mathrm{T} 7$ and $\mathrm{T} 3$ were created by direct genome manipulation in yeast [42]. Here both T3 with T7-like gp17 and T7 with T3-like gp17 were generated, and these showed the expected changes in plating efficiencies on different strains of $E$. coli. The work was then expanded into modification of T3 gpl7 to match the sequence of $g p 17$ from the $Y$. pseudotuberculosis phage $\mathrm{R}$, which expanded the T3 host-range to match that of phage $\mathrm{R}$ whilst still retaining the lytic function of $\mathrm{T} 3$ and its wild-type ability to infect $E$. coli. Finally, the transfer of the gp17 from T7-like Klebsiella phage K11 into T7 highlighted the interesting compatibility issues that can arise from the transfer of tail fiber genes between bacteriophages. In this case it was not possible to generate infectious $\mathrm{T} 7$ phage with $g p 17$ from K11. It appears that this is due to incompatibility between tail fibers of K11 and the 'collar' assembly that mediates attachment of the tail fibers to the head of the bacteriophage. This meant that generating a modified $\mathrm{T} 7$ with the ability to infect Klebsiella required not only the gp17 gene of K11, but also replacement of the 'collar' genes $g p 11$ and $g p 12$ with those from K11. However, once the both 'collar' and tail fiber genes had been altered, this newly modified T7 phage was able to infect and kill Klebsiella, suggesting that the modified $\mathrm{T} 7$ retained its lytic properties even after shifting hosts from E. coli [42].

Of course, attachment and genome delivery is only the first stage at which phages interact with the host; lysogeny or lytic replication and assembly of phage progeny will require interactions with a huge variety of host proteins and pathways to complete the life-cycle. To give an example, an extensive number of known interactions between the phage lambda and $E$. coli have been reviewed 
by Friedman [43]. Therefore, it is likely that to a certain degree the bacteriophage genome will become specialised in order to mediate host-specific interactions, for example within the lambda-like family of $E$. coli tailed-phages there are cases where phages show a convincing genome mosaic relationship to the lambda-family, but with altered species specificity and highly divergent nucleotide sequences $[44,45]$. Therefore in the future more fundamental work is required to investigate the underlying mechanisms of phage-host interactions in order to better understand the ways in which the phage genome may adapt at other stages of its life-cycle. Although direct manipulation of these interactions through traditional genetic engineering may be difficult, modern synthetic biology methodologies such as directed evolution, may allow us to rapidly adapt and modify phage to a desired host in vitro $[46,47]$. The ability to modify or control these interactions may play a key role in our ability to engineer phage specificity beyond control of attachment and entry.

\section{ENGINEERING PHAGE LONGEVITY in vitro AND MINIMISING NEGATIVE PATIENT-PHAGE INTERACTIONS}

Utilising phages in a clinical setting presents challenges that are not typically relevant to drug-based approaches. For example, how can the patient immune response to phages be minimised and how can phage persistence in patients be increased to maximise the potential for infection of the bacterial target. In addition, there are also concerns regarding the clinical effects of the induction of bacterial lysis by phages, which may release large amounts of bacterial substances such as lipopolysaccharide (LPS/endotoxin) or other pathogen-associated molecular patterns (PAMPS) into the phage treated patient. Release of PAMPs such as endotoxins from gram-negative bacteria could have immediate adverse effects on the phage treated patient by stimulating acute and systemic inflammatory immune responses [48] and phage therapy trials in humans have made observational links between endotoxin release and reports of negative post-treatment side-effects [49]. However, the immune modulation effects of both endotoxin release and the application of phage could conceivably also have positive effects on patient outcome, and therefore there may be an element of risk and reward when treating bacterial infections with lytic phages and, as with all therapeutics, a need to balance potential toxicity against maximising positive patient outcomes. It is also necessary to identify whether phage isolated and considered for therapeutic use contain any factors that act to enhance bacterial virulence upon infection, with an extreme example being the Shiga toxin-encoding phages of the lambda-family [50]. Utilising phages as antimicrobial agents therefore may not be as simple as choosing a combination of strongly lytic phages, or phages with a particularly useful host-range. These considerations have led to the engineering of phages to improve their bioavailability and to alter their methods of killing bacteria becoming an active area of research. This is further compounded by the safety concerns with using a replicative and genetically plastic virus in a healthcare context, as there is no guarantee that a replicative phage delivered to the patient will be genetically stable in vivo or whether it will lead to uncontrolled onward transmission from the treated patient unless serious considerations have been given to genetic manipulation of the phage to address these risk factors [51].

The most well-defined immune response to phage is that of virus neutralising $(\mathrm{VN})$ antibodies targeted against tail fibers. Binding of anti-tail antibodies to phage inhibits virus binding to bacteria, and can render a phage population entirely non-functional by reducing the availability of unbound infective phages in the patient $[52,53]$. However, this is certainly not the only immune response to phage, and Sokoloff et al. have shown that modification of phage capsids by addition of C-terminal lysine or arginine residues aids the phage in escaping complement-mediated inactivation by binding C-reactive protein [54]. Another study by Merril et al. utilised the classical virological method of modification/adaptation by serial passage to isolate a lambda phage population that was able to avoid the murine immune response, specifically the action of the mononuclear phagocyte system that typically leads to phage clearance [55]. Analysis of two of these 'long-circulating' mutants identified a common mutation in the major capsid protein $\mathrm{E}$ which increased phage concentrations in the blood at 18 hours post inoculation approximately 10,000-fold; subsequent work then confirmed that transfer of this single mutation back into the wild-type lambda phage conferred the full 'long-circulating' phenotype $[55,56]$. Similar results were obtained by Capparelli et al. who found that serially passaged mutants of an environmental isolate of an E. coli O157:H7 phage and a Staphylococcus aureus phage were able to persist in the circulation up to 10 times longer (up to one month post-inoculation) than the wild-type and, interestingly, did not induce the formation of neutralizing antibodies [57,58]. A study by Łusiak-Szelachowska et al. has analysed the presence of phage neutralising antibodies in human patients undergoing phage therapy, and found that whilst the level of antibody stimulation was inconsistent among the group, $12.3 \%$ of patients exhibited a strong phage neutralising response upon treatment, although this did not exclude a positive patient outcome [59]. It therefore may not be necessary to engineer phage such as to reduce the antiphage immune response in all cases, and could depend on the type of phage (replicative or non-replicative), the 
specific bacterial infection to be treated, route of administration, and planned number of phage administrations per treatment. Minimising this response could also be important in the context of the goals of producing an engineered broad-spectrum phage as a therapeutic. If a single phage is identified for use as a scaffold into which broad host-range efficacy can be engineered, it may be reasonable to consider that this phage could subsequently be used for multiple treatments of bacterial disease across a patient's life. In this case minimising immune reactivity to the phage scaffold could be vital to maintaining efficacy upon re-administration.

A key issue in the field is the potential risk to the patient when treating bacteria with lytic phage which causes the release of endotoxins upon large-scale bacterial lysis, as outlined in reviews by Abedon et al. and Loc-Carillo \& Abedon $[4,60]$. Thankfully this is a problem to which our increasing ability to modify and engineer structural and regulatory components of the phage genome in order to alter the phage life-cycle or deliver synthetic genes is well suited. Through the modification of lysogenic phages to become toxic but remain non-lytic, or lytic phages to become non-lytic but retain toxicity, it appears to be possible to alleviate risks of endotoxin release. Hagens and Bläsi demonstrated that the small non-lytic phage M13 can be engineered to express one of two lethal proteins upon infection [61]. Both engineered M13 phages expressing the $R$-gene from the $B g l I I$ restrictionmodification system of Bacillus globigii and a lambdaderived holin were able to kill $99 \%$ of infected bacteria, with endotoxin levels approximately 4-fold lower after lysis when compared to a virulent lambda control. However, a 99\% killing rate appears to have been insufficient to prevent rapid re-growth of a bacterial population that was resistant to subsequent infection by the modified M13 phage. A similar methodology to the above was used to develop a non-lytic and non-replicative $P$. aeruginosa phage Pf3R, but in this case an essential transport protein from ORF40 of Pf3 was truncated and the $R$-gene of the BglII system added downstream, rendering Pf3R non-replicative but toxic to bacteria not harbouring the protective BglII methylase ( $M-$-) gene [62]. This study used in vivo testing of both wild-type Pf3 and $\mathrm{Pf3R}$ in a murine peritonitis infection model and provided data showing that both Pf3 and Pf3R are effective in treating $P$. aeruginosa infection, with $\mathrm{Pf} 3 \mathrm{R}$ improving percentage of survival when higher inoculating doses of bacteria were used. This result confirms expectations that the amount of endotoxin released following Pf3 lysis is increased at high bacterial cell number, highlighting the negative effects of lysis by the wild-type phage and the difference in method of action between Pf3 and Pf3R. An independent confirmation of the potential importance of using a non-lytic killing phage in vivo was provided by
Matsuda et al. who analysed the properties of the lysisdeficient T4 mutant T4LyD in comparison to wild-type T4 in a murine peritonitis model [63]. T4LyD contains an amber mutation in the holin gene, producing a truncated, non-functional holin, which ablates the ability of the phage to lyse cells at the final stage of replication. Treatment of mice in an E. coli peritonitis model using the $\mathrm{T} 4 L y D$ phage significantly improved survival compared to untreated mice and mice treated with wild-type $\mathrm{T} 4$, with levels of inflammatory mediators such as tumor necrosis factor alpha (TNF- $\alpha$ ) and interleukin-6 (IL-6) reduced 2-fold in $\mathrm{T} 4 L y D$ treated mice compared to controls [63].

In a different non-lytic and non-replicative killing strategy Westwater et al. utilised an M13-based phagemid system to deliver a plasmid capable of inducible overexpression of the toxic components of natural $E$. coli conditionally lethal genes [64]. The benefits of the phagemid system are significant in the context of addressing some potential pitfalls of phage therapy. In order to derive a phagemid, the necessary genes for genome amplification, capsid assembly and packaging, and host lysis are supplied in trans, so whilst the phagemid plasmid is packaged into a functional infectious phage particle, this particle lacks multiple components that are essential for onwards replication (Figure 2). This system therefore prevents uncontrolled release of progeny phages into the surrounding environment, and avoids non-specific disruption of the bacterial cell wall and subsequent release of endotoxins caused by the release of the progeny phages. Westwater et al. showed that infection of $E$. coli with M13 phagemids containing either the gef or chpBK genes under control of the LacI promoter led to a $99.99 \%$ drop in cell viability within 2 hours of IPTG induction [64]. Further work using M13 phagemids has yielded phages that are capable of stimulating a lethal uncoupling of catabolism in pathogenic $E$. coli $\mathrm{O} 157: \mathrm{H} 7$ by expressing a modified catabolic activator protein (CAP) [65]. This paper also highlights the importance of considering multiplicity of infection (MOI) when utilising a phagemid system that is incapable of replication in vivo, as killing efficiency dropped dramatically when infections were carried out with an MOI below 10, which is supported in a study carried out by Kasman et al. that has experimentally validated a model of how observed MOIs correspond to theoretical MOIs in M13 and P1 phagemid infections [66]. Most recently, Krom et al. have further extended the range of non-lytic antimicrobial peptides that have been tested using the M13 phagemid system [67]. The most efficacious phagemid vector expressed the antimicrobial peptides cecropin and apidaecin in combination with the bacterial toxin $\mathrm{CcdB}$, and significantly improved survival in a murine peritonitis infection model. Interestingly, 


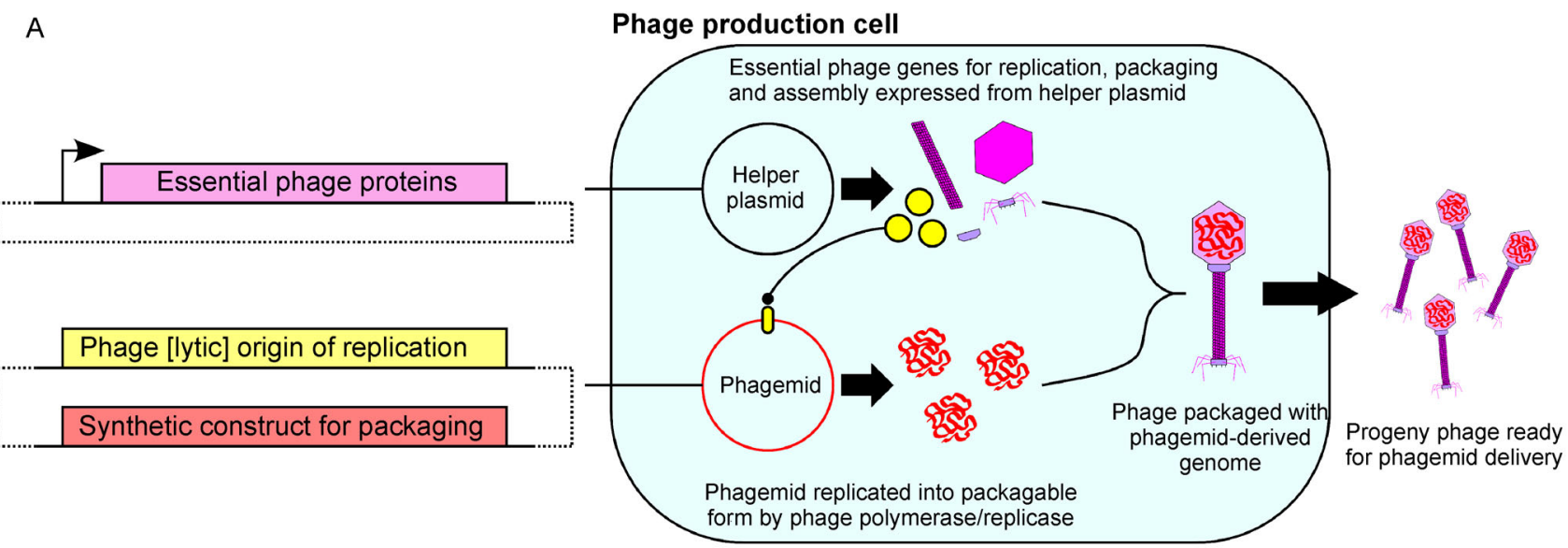

B
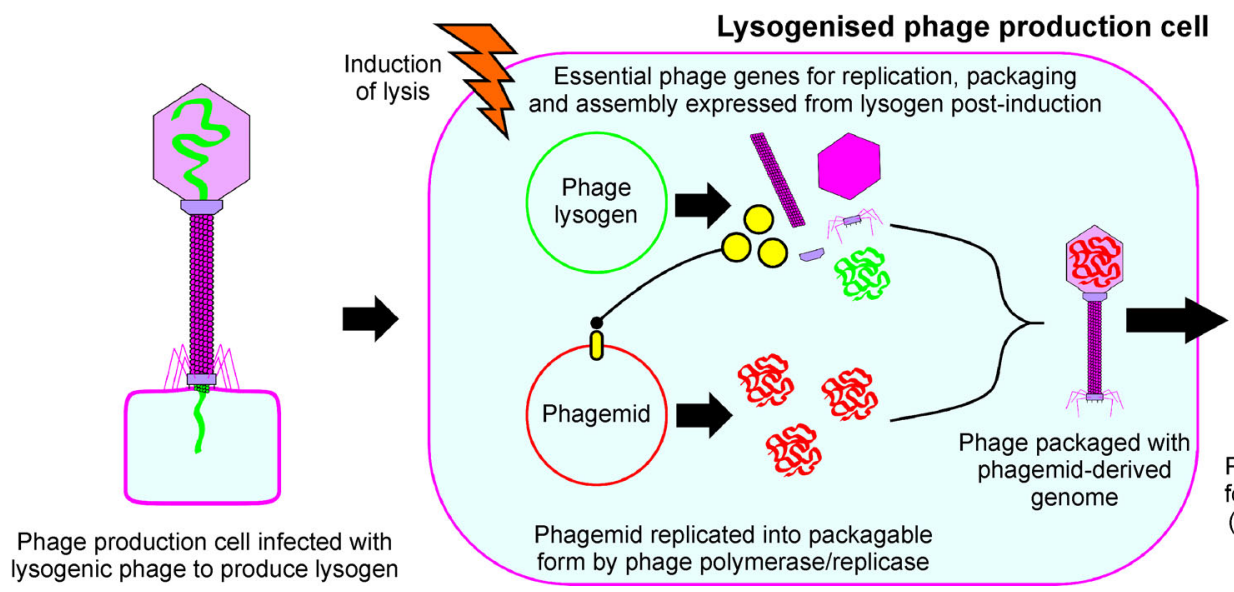

Lysogenised phage production cell lysogenic phage to produce lysogen

form by phage polymerase/replicase

Figure 2. Schematics showing the two methods for phagemid packaging into infectious phage particles. In (A) helper plasmid is co-transformed into the phage production cell along with the phagemid. This helper plasmid is capable of expressing all essential genes necessary for phage assembly, replication of the phagemid into a form that can be packaged into the phage capsid, and exit of progeny phages from the host. Therefore upon co-transformation the production cell is capable of [often inducible] production of progeny phages packaged with the phagemid ready for purification and subsequent infections. In (B) the phage production cell is first lysogenised with a lysogenic phage. This lysogen can subsequently be transformed with the phagemid construct, and contains the entire phage genome. Upon induction of lysis, the lysogen will begin to express these genes, and concurrently amplify and package both the lysogen DNA and the phagemid into progeny phage. This mixed population of lysogenic and phagemid-packaged progeny phages will be released upon completion of lysis, and can subsequently be purified and used in subsequent infections.

infection of mice with M13 phagemid not containing any antimicrobial peptides or toxin did appear to have a positive effect on mouse survival, although this effect was not statistically significant when compared to untreated controls. It therefore may be interesting to consider further investigation of whether the M13 delivery system is capable of promoting host defence even in the absence of a toxic payload, possibly by recruiting immune cells to the site of bacterial infection, or whether M13 attachment and phagemid injection has other subtle effects on bacterial viability in vivo.

\section{UTILISING BACTERIOPHAGES TO ENGINEER BACTERIAL TREATMENT SUSCEPTIBILITY AND MODIFY THE BACTERIAL GENOME}

The huge success and efficacy of treatment of bacterial disease with antibiotic drugs is unprecedented, with the ubiquitous nature of bacterial interaction with human and animal health meaning that their impact on healthcare has been immense. The broad-spectrum efficacy of antibiotics 
is however both a gift, with healthcare practitioners able to choose medication in the knowledge they will treat disease even without a specific bacterial diagnosis, and a curse, with a large pool of susceptible bacteria all undergoing strong selection to escape drug action and acquire resistance upon antibiotic treatment [6]. Horizontal gene transfer between bacteria also means that any emerging resistance mutation of mobile genetic element conferring resistance can be rapidly disseminated amongst various bacterial species. Another important effect of antibiotic treatment is that disruption of commensal flora may predispose the patient to secondary infection, as more robust or treatment-resistant pathogenic bacteria can rapidly fill empty niches left by killed commensal bacteria [68]. These observations have led to two major avenues of investigation in the context of bacteriophage therapies (Figure 3). Firstly is it possible to utilise phages with broad host specificity that subsequently show a degree of strain-specificity in their action of killing? Secondly, can phages be used to modify the genomes of bacteria such that they generate susceptibility to subsequent treatment, possibly by employing such a strategy as part of a combination treatment strategy?

With flexible packaging limits and promiscuity in terms of genome packaging, bacteriophages have been identified as an ideal carrier to deliver foreign genes and constructs into bacteria in situations where direct transformation is impossible. The rapid development of sequence specific RNA-guided nucleases such as the CRISPR-Cas9 system [69] has also meant that phagemediated genome-specific targeting of bacteria is becoming a realistic goal, with two major publications produced in this area. Firstly, Citorik et al. used CRISPR targeting RNAs (crRNA(s)) to enable the Cas9 endonuclease to restore carbenicillin susceptibility to resistant E. coli, target DNA gyrase (gyrA) mutants resistant to quinolone antibiotics, and also delete an intimin-encoding chromosomal virulence gene eae from Enterohaemorrhagic E. coli (EHEC) O157:H7 [70]. Delivery was achieved using an M13 phagemid system, with a high transduction and cutting efficiency of between $99 \%$ and $99.99 \%$ observed in vitro. In the case of cutting the episomal resistance plasmid pNDM-1, the presence of a plasmidborne toxin-antitoxin system, by which stability of episomal elements can be enhanced by post-segregational killing of daughter cells lacking the plasmid, appears to have led to selective killing of bacteria after phagemid delivery due to plasmid loss rather than antibiotic resensitisation. The effect of eae disruption was also tested in a Galleria mellonella larval infection model to investigate whether phage treatment of infected $G$. mellonella larvae correlates leads to decreased bacterial pathogenicity in vivo. In this case, whilst there was a significant difference in survival between the larvae treated with eae-targeting phagemid compared to the untreated and non-targeting control, survival was not enhanced to a large degree. This may again highlight the difficulties in achieving a high MOI and infection rate in vivo and how this can limit the efficacy of non-replicative phage treatments. The second major publication, by Bikard et al., used chromosome and episome-specific crRNA targeting to selectively kill antibiotic-resistant Staphylococcus aureus strains [71]. A staphylococcal phagemid delivery system was developed based on the phage $\Phi N M 1$, with the phagemid carrying the Cas9 endonuclease together with crRNAs targeting either the chromosomally-located aph-3 kanamycin resistance gene, the methicillin resistance gene mecA in the MRSA strain USA $300^{\Phi}$, or the USA300 virulence plasmids pUSA01 and pUSA02. When treating the aph-3 carrying $S$. aureus with the $\Phi$ NM1 phagemid in vitro, reduction in viable cell counts by $99.99 \%$ was observed. When targeting the MRSA strain USA $300^{\Phi}$ and its virulence plasmids pUSA01 and pUSA02, reductions in cell viability under selective conditions (the presence of oxacillin or tetracycline respectively) were observed to be between $99 \%-99.9 \%$. Interestingly, the authors also considered the use of phagemid as a mechanism of bacterial immunisation, with RN4220 cells treated with the mecA-targeting construct unable to subsequently inherit the pUSA02 virulence plasmid. An in vivo test of the aph-3 targeting efficacy of the $\Phi$ NM1 phagemid was carried out using a mouse skin colonisation model. In this case reduction in numbers of viable aph-3 positive cells was not reduced to a similar degree as observed in in vitro studies, with a $77 \%$ reduction in the numbers of viable kanamycin-resistant bacteria after in vivo treatment. However, this was significantly different to other experimental conditions, showing that the phagemid is capable of infection and sequence-specific gene targeting in vivo. A study by Yosef et al. has modified the genome of lambda phage to include a type I-E CRISPR system along with spacer sequences targeting, and thus preventing transformation of, lambda-lysogenised E. coli with antibiotic resistance plasmids [72]. Interestingly, they also considered methods by which maintenance of this modified lambda lysogen could confer a selective advantage to the lysogenised E. coli. By adding protospacers targeting synthetic sequences within a modified lytic phage, they were able to confer resistance to phage infection to the lysogen, meaning that the proportion of modified lysogens within a mixed bacterial population could be enriched through infection with the lytic phage, with lysogens showing enhanced resistance to lysis compared to wild-type bacteria. The leveraging of sequence-specific targeting in phage-mediated treatment of bacterial disease is still at a very early stage in terms of testing feasibility and efficacy. However, it does offer an 


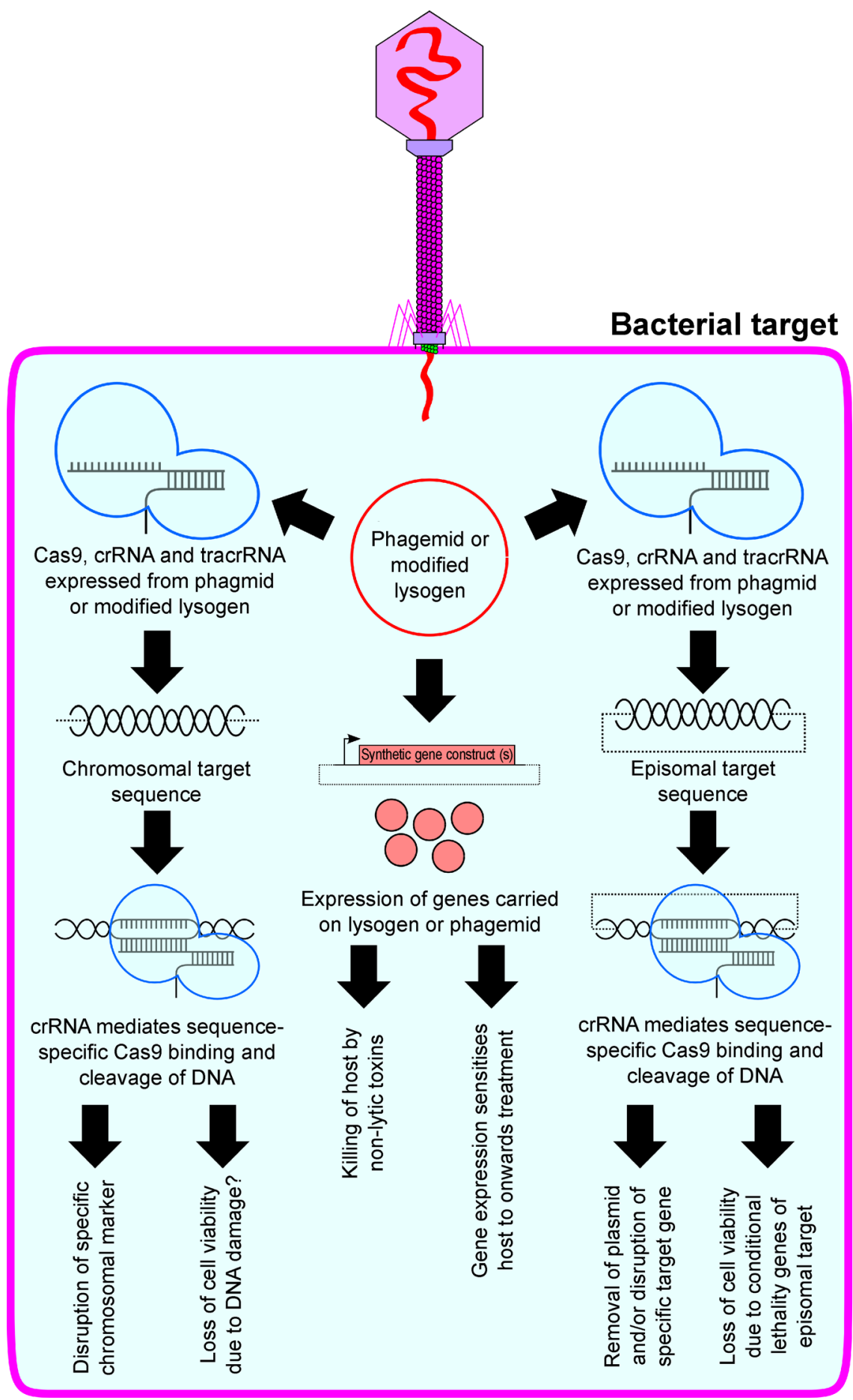

Figure 3. Schematics summarizing methods for modification of the host genome and engineering altered bacterial sensitivity used for onwards treatment after infection with a genetically engineered phage. Genes can be introduced to infected bacteria in the form of a modified lysogen or a phagemid vector. Expression of genes from these sources can either be used to directly kill bacteria, for example by using conditional lethality genes from toxin-antitoxin systems, or to sensitise the bacteria to onwards treatment such as with dominant sensitive genes. A second option is to introduce a gene editing system such as CRISPRCas9, where upon phagemid or lysogen entry, genetic markers can be specifically targeted and thereby inactivated. This allows for the killing or modification of bacteria in a sequence-dependent manner, minimising the risks of disruption in non-targeted commensal bacterial populations. 
interesting alternate approach to traditional bacteriophage treatments, especially in the context of phagemid delivery systems. Here it is possible to imagine that concerns regarding host-specific interactions at the level of phage replication could be mitigated by the use of a nonreplicative system which also offers control over such features as broad-spectrum origins of replication and promoters at the level of phagemid design. This could leave researchers free to concentrate on methods of increasing host-range and infection efficiency through modification of features associated with attachment and injection, which as discussed above is a key consideration that the field is currently investigating.

Whilst the body of evidence regarding phage therapies is still expanding and developing, it appears that at this stage the ability to utilise phages to reach a level of efficacy seen when using antibiotic drugs is beyond our current level of understanding. This conclusion had led to the investigation of whether phages can also be useful as part of combination therapies with traditional antimicrobials to enhance sensitivity or re-introduce sensitivity into strains of bacteria which have become resistant to current treatment regimes. A number of different approaches have been considered regarding how phages can best be utilised as part of combination therapies (Figure 3). Yacoby et al. have chemically modified filamentous phages to specifically deliver antibiotic drugs to Staphylococcus, Streptococcus and E.coli bacteria [73]. Although strictly speaking this is a chemical rather than a genetic engineering approach to the problem, by combining anti-bacterial polyclonal IgG antibodies bound to the minor phage coat protein p3 with chloramphenicol chemically attached to the major coat protein $\mathrm{p} 8$ it was possible to increase the potency of the antibiotic 10,000-fold compared to treatment with chloramphenicol alone. A more relevant approach in the context of this review was taken by Lu and Collins. Here the genome of M13 phage was modified to deliver the lexA3 gene, which encodes a repressor of the bacterial SOS DNA repair system [74] and increases the sensitivity of bacteria to antibiotics such as quinolones that cause DNA damage as part of their antimicrobial action (as topoisomerase LT inhibitors). Upon infection with the modified M13 phage, sensitivity to ofloxacin was increased between 2.7 - to 4.5 -fold. The phage-mediated enhancement of ofloxacin efficacy was also tested in vivo in a murine intraperitoneal infection model. Here an increase in mouse survival from $20 \%$ to $80 \%$ was observed in mice treated with both the engineered phage and ofloxacin when compared to ofloxacin treatment alone. Interestingly, the difference in survival rates between treatment with wild-type phage and ofloxacin compared to engineered phage and ofloxacin was reduced to $30 \%$, indicating that wild-type phage alone had a potentiating effect on the antibiotic efficacy. In a similar study, Edgar et al. modified the genome of lambda phage to introduce the antibiotic sensitising genes $r p s L$ and gyrA, which are expressed upon lysogeny [75]. When antibiotic resistant $E$. coli mutants were lysogenised with the modified lambda phage, antibiotic sensitivity was restored, with minimum inhibitory concentrations of streptomycin and nalidixic acid decreasing 8- and 2fold respectively. As a side point from this paper, considerations were also given as to how best to allow selection for the modified lysogen without introducing an additional antibiotic selection marker. This is a key consideration when using phage to remove resistance genes, as removal of one marker cannot be traded for the introduction of another. Here overexpression of the teh $A B$ gene from a $\mathrm{T} 7$ promoter, which confers resistance to the toxic tellurium compound tellurite, was successfully substituted for a chloramphenicol resistance cassette. Finally, a study has also investigated the use of phagemidmediated RNA silencing to reduce transcription from antibiotic resistance genes [76]. Unfortunately, it appears that the RNA silencing of resistance genes is not a highlyefficient process, with only $29 \%$ of bacteria positive for the phagemid showing increased sensitivity to the antibiotic. When compared to more recent work on cas9-mediated gene targeting, this appears to be a more challenging avenue of investigation.

\section{CONCLUSIONS}

The goal of this article was to highlight key examples from the literature on how bacteriophages have been modified to increase their utility as antimicrobials in a healthcare context. The combination of improved tools for genome analysis and modification along with the increasing threat of widespread antibiotic resistance amongst diverse microbes has led to a long-overdue resurgence of phage research. Whilst worries regarding the efficacy, safety and usefulness of phage therapies do remain, these problems are now being investigated, and with modern tools it may finally be possible to address these concerns. Although there may not be one obvious single solution attempts to combine research insights from multiple areas into a single phage backbone may yield exciting results.

Since biological containment and control is an important consideration, especially in the context of genetically engineered organisms and viruses, it appears that non-replicative phagemid systems may offer an ideal opportunity to address these issues, with the M13 phagemid system being especially well suited to delivering promising results. The phagemid system also opens the possibility of rescuing phages from highly-modified specialised lysogens or helper-plasmids, which could 
make the manipulation of phage components such as tail fibres and capsids easier to achieve, whilst also offering complete control over the genetic material delivered upon phage infection. However, this enthusiasm must be tempered by the fact that non-replicative systems may make it extremely difficult to achieve a therapeutic dose of phages in vivo.

Despite recent efforts, the issues of application, delivery and control are still relevant to both genetically modified and wild-type phage, and as such these are currently likely to be more useful for topical site-specific treatments such as surgical wounds and burns, or as a method for preventing bacterial infection at interfaces between biological and material surfaces. However, the consideration of phage use as a more broad-spectrum treatment should not be discounted, with emerging possibilities for modifying and removing deleterious marker sequences from a large bacterial population without the need for a general bacterial killing mechanism being an enticing prospect. There have been a number of recent examples that the bacterial CRISPR system can be effectively integrated into both phage and phagemid genomes, and although there may be numerous challenges in utilising phages to significantly modify the vast bacterial populations found in both animals and humans, the opportunities for control and targeted removal of genetic features in bacteria are exciting.

Overall, the recent resurgence of interest in phages, and the ways in which we may be able to control and modify their properties and behaviour as antimicrobials, is of great interest and potential importance. There have been a number of exciting developments in the field, with only a selection of these developments highlighted in this review. There are significant differences between even a non-replicative phagemid and an antimicrobial drug treatment; how these differences are reconciled within the current pharmaceutical testing, licensing and 'Good Manufacturing Practices' (GMP) framework will be interesting and possibly significantly impact the direction of future applied phage research [36,77]. Also, many of the phage engineering solutions discussed here would give rise to what would be considered a geneticallymodified organism (GMO) and as such the licensure and clinical use of these phage would be tightly regulated. There has been some review of the safety and licencing GMO's in the context of bacterial vaccines [78] and replicative viral vaccines derived from recombinant DNA, such as the live-attenuated Influenza A and B vaccine Flumist ${ }^{\circledR} /$ Fluenz $^{\circledR}$, are currently licenced for use in the US [79] and Europe [80]. Therefore whilst extra considerations must be made, such as the potential transmissibility and dissemination of a GMO phage beyond the treated patient and the ability of the modified features to transfer to naturally-occurring phages, this may not be an absolute limitation on their potential therapeutic use. Hopefully continued research support in this area will allow the field to continue to address concerns regarding phage therapies, and finally unlock their significant potential as antimicrobial agents to enhance human and animal health.

\section{ACKNOWLEDGMENTS}

This work was supported by the Bill and Melinda Gates Foundation under the Grand Challenges Explorations grant (OPP1139488).

\section{COMPLIANCE WITH ETHICS GUIDELINES}

The authors Russell Brown, Andreas Lengeling and Baojun Wang declare that they have no conflict of interests

This article does not contain any studies with human or animal subjects performed by any of the authors.

\section{OPEN ACCESS}

This article is distributed under the terms of the Creative Commons Attribution 4.0 International License (http://creativecommons.org/licenses/ by/4.0/), which permits unrestricted use, distribution, and reproduction in any medium, provided you give appropriate credit to the original author(s) and the source, provide a link to the Creative Commons license, and indicate if changes were made.

\section{REFERENCES}

1. Summers, W. C. (2012) The strange history of phage therapy. Bacteriophage, 2, 130-133

2. Twort, F. W. (1915) An investigation on the nature of ultra-microscopic viruses. Lancet, 186, 1241-1243

3. d'Herelle, F. (1917) On an invisible microbe antagonistic to dysentery bacili. CR Acad. Sci. Paris, 165, 373-375

4. Abedon, S. T., Kuhl, S. J., Blasdel, B. G. and Kutter, E. M. (2011) Phage treatment of human infections. Bacteriophage, 1, 66-85

5. Neu, H. C. (1992) The crisis in antibiotic resistance. Science, 257, 1064-1073

6. Davies, J. and Davies, D. (2010) Origins and evolution of antibiotic resistance. Microbiol. Mol. Biol. Rev., 74, 417-433

7. Bradley, R. W., Buck, M. and Wang, B. (2016) Tools and principles for microbial gene circuit engineering. J. Mol. Biol., 428, 862-888

8. Wang, B. and Buck, M. (2012) Customizing cell signaling using engineered genetic logic circuits. Trends Microbiol., 20, 376-384

9. Wang, B., Kitney, R. I., Joly, N. and Buck, M. (2011) Engineering modular and orthogonal genetic logic gates for robust digital-like synthetic biology. Nat. Commun., 2, 508

10. Wang, B., Barahona, M. and Buck, M. (2013) A modular cell-based biosensor using engineered genetic logic circuits to detect and integrate multiple environmental signals. Biosens. Bioelectron., 40, 368-376

11. Bradley, R. W. and Wang, B. (2015) Designer cell signal processing circuits for biotechnology. N. Biotechnol., 32, 635-643

12. Haellman, V. and Fussenegger, M. (2016) Synthetic biology - toward therapeutic solutions. J. Mol. Biol., 428, 945-962

13. Smith, H. O., Hutchison, C. A. III, Pfannkoch, C. and Venter, J. C. 
(2003) Generating a synthetic genome by whole genome assembly: ФX174 bacteriophage from synthetic oligonucleotides. Proc. Natl. Acad. Sci. USA, 100, 15440-15445

14. Chan, L.Y., Kosuri, S., and Endy, D. (2005) Refactoring bacteriophage T7. Mol. Syst. Biol. 1, 2005. 0018

15. Lu, T. K. and Koeris, M. S. (2011) The next generation of bacteriophage therapy. Curr. Opin. Microbiol., 14, 524-531

16. Pires, D. P., Cleto, S., Sillankorva, S., Azeredo, J. and Lu, T. K. (2016) Genetically engineered phages: a review of advances over the last decade. Microbiol. Mol. Biol. Rev., 80, 523-543

17. Rakhuba, D. V., Kolomiets, E. I., Dey, E. S. and Novik, G. I. (2010) Bacteriophage receptors, mechanisms of phage adsorption and penetration into host cell. Pol. J. Microbiol., 59, 145-155

18. Crawford, J. T. and Goldberg, E. B. (1977) The effect of baseplate mutations on the requirement for tail-fiber binding for irreversible adsorption of bacteriophage T4. J. Mol. Biol., 111, 305-313

19. Crawford, J. T. and Goldberg, E. B. (1980) The function of tail fibers in triggering baseplate expansion of bacteriophage T4. J. Mol. Biol., 139, 679-690

20. Arscott, P. G. and Goldberg, E. B. (1976) Cooperative action of the T4 tail fibers and baseplate in triggering conformational change and in determining host range. Virology, 69, 15-22

21. Molineux, I. J. (2001) No syringes please, ejection of phage T7 DNA from the virion is enzyme driven. Mol. Microbiol., 40, 1-8

22. Kemp, P., Garcia, L. R. and Molineux, I. J. (2005) Changes in bacteriophage $\mathrm{T} 7$ virion structure at the initiation of infection. Virology, 340, 307-317

23. Heller, K. and Braun, V. (1979) Accelerated adsorption of bacteriophage T5 to Escherichia coli F, resulting from reversible tail fiberlipopolysaccharide binding. J. Bacteriol., 139, 32-38

24. Heller, K. and Braun, V. (1982) Polymannose O-antigens of Escherichia coli, the binding sites for the reversible adsorption of bacteriophage $\mathrm{T}^{+}$via the L-shaped tail fibers. J. Virol., 41, 222227

25. Riede, I., Degen, M. and Henning, U. (1985) The receptor specificity of bacteriophages can be determined by a tail fiber modifying protein. EMBO J., 4, 2343-2346

26. Montag, D., Riede, I., Eschbach, M.-L., Degen, M. and Henning, U. (1987) Receptor-recognizing proteins of T-even type bacteriophages: constant and hypervariable regions and an unusual case of evolution. J. Mol. Biol., 196, 165-174

27. Moody, M. F. (1973) Sheath of bacteriophage T4: III. contraction mechanism deduced from partially contracted sheaths. J. Mol. Biol., 80, 613-635

28. Wright, A., Hawkins, C. H., Änggård, E. E. and Harper, D. R. (2009) A controlled clinical trial of a therapeutic bacteriophage preparation in chronic otitis due to antibiotic-resistant Pseudomonas aeruginosa; a preliminary report of efficacy. Clin. Otolaryngol., 34, 349-357

29. Gu, J., Liu, X., Li, Y., Han, W., Lei, L., Yang, Y., Zhao, H., Gao, Y., Song, J., Lu, R., et al. (2012) A method for generation phage cocktail with great therapeutic potential. PLoS One, 7, e31698

30. Oliveira, A., Sereno, R. and Azeredo, J. (2010) In vivo efficiency evaluation of a phage cocktail in controlling severe colibacillosis in confined conditions and experimental poultry houses. Vet. Microbiol., 146, 303-308

31. Jaiswal, A., Koley, H., Ghosh, A., Palit, A. and Sarkar, B. (2013) Efficacy of cocktail phage therapy in treating Vibrio cholerae infection in rabbit model. Microbes Infect., 15, 152-156
32. Chan, B. K. and Abedon, S. T. (2012). Chapter 1 - Phage therapy pharmacology: phage cocktails. In Advances in Applied Microbiology, Laskin, A.I., Sariaslani, S. and Gadd, G.M. ed. 1-23. Massachusetts: Academic Press

33. Chan, B. K., Abedon, S. T. and Loc-Carrillo, C. (2013) Phage cocktails and the future of phage therapy. Future Microbiol., 8, 769-783

34. Gill, J. J. and Hyman, P. (2010) Phage choice, isolation, and preparation for phage therapy. Curr. Pharm. Biotechnol., 11, 2-14

35. Merabishvili, M., Pirnay, J.-P., Verbeken, G., Chanishvili, N., Tediashvili, M., Lashkhi, N., Glonti, T., Krylov, V., Mast, J., Van Parys, L., et al. (2009) Quality-controlled small-scale production of a well-defined bacteriophage cocktail for use in human clinical trials. PLoS One, 4, e4944

36. Kutter, E., De Vos, D., Gvasalia, G., Alavidze, Z., Gogokhia, L., Kuhl, S. and Abedon, S. T. (2010) Phage therapy in clinical practice: treatment of human infections. Curr. Pharm. Biotechnol., 11, 69-86

37. Mahichi, F., Synnott, A. J., Yamamichi, K., Osada, T. and Tanji, Y. (2009) Site-specific recombination of T2 phage using IP008 long tail fiber genes provides a targeted method for expanding host range while retaining lytic activity. FEMS Microbiol. Lett., 295, 211-217

38. Yoichi, M., Abe, M., Miyanaga, K., Unno, H. and Tanji, Y. (2005) Alteration of tail fiber protein gp38 enables T2 phage to infect Escherichia coli O157:H7. J. Biotechnol., 115, 101-107

39. Pouillot, F., Blois, H. and Iris, F. (2010) Genetically engineered virulent phage banks in the detection and control of emergent pathogenic bacteria. Biosecur. Bioterror., 8, 155-169

40. Krüger, D. H. and Schroeder, C. (1981) Bacteriophage T3 and bacteriophage T7 virus-host cell interactions. Microbiol. Rev., 45, 9-51

41. Lin, T. -Y., Lo, Y. -H., Tseng, P. -W., Chang, S. -F., Lin, Y. -T. and Chen, T. -S. (2012) A T3 and T7 recombinant phage acquires efficient adsorption and a broader host range. PLoS One, 7, e30954

42. Ando, H., Lemire, S., Pires, D. P. and Lu, T. K. (2015) Engineering modular viral scaffolds for targeted bacterial population editing. Cell Syst., 1, 187-196

43. Friedman, D. I. (1992) Interaction between bacteriophage $\lambda$ and its Escherichia coli host. Curr. Opin. Genet. Dev., 2, 727-738

44. Casjens, S. R., Gilcrease, E. B., Winn-Stapley, D. A., Schicklmaier, P., Schmieger, H., Pedulla, M. L., Ford, M. E., Houtz, J. M., Hatfull, G. F. and Hendrix, R. W. (2005) The generalized transducing Salmonella bacteriophage ES18: complete genome sequence and DNA packaging strategy. J. Bacteriol., 187, 1091-1104

45. Casjens, S. (2003) Prophages and bacterial genomics: what have we learned so far? Mol. Microbiol., 49, 277-300

46. Esvelt, K. M., Carlson, J. C. and Liu, D. R. (2011) A system for the continuous directed evolution of biomolecules. Nature, 472, 499-503

47. Bassalo, M. C., Liu, R. and Gill, R. T. (2016) Directed evolution and synthetic biology applications to microbial systems. Curr. Opin. Biotechnol., 39, 126-133

48. Prins, J. M., van Deventer, S. J., Kuijper, E. J. and Speelman, P. (1994) Clinical relevance of antibiotic-induced endotoxin release. Antimicrob. Agents Chemother., 38, 1211-1218

49. Slopek, S., Durlakowa, I., Weber-Dabrowska, B., KucharewiczKrukowska, A., Dabrowski, M. and Bisikiewicz, R. (1983) Results of bacteriophage treatment of suppurative bacterial infections. I. General evaluation of the results. Arch. Immunol. Ther. Exp. (Warsz.), 31, 267291

50. Gamage, S. D., Patton, A. K., Hanson, J. F. and Weiss, A. A. (2004) Diversity and host range of Shiga toxin-encoding phage. Infect. 
Immun., 72, 7131-7139

51. Krylov, V. N. (2001) Phagotherapy in terms of bacteriophage genetics: hopes, perspectives, safety, limitations. Genetika, 37, 869-887

52. Jerne, N. K. and Avegno, P. (1956) The development of the phageinactivating properties of serum during the course of specific immunization of an animal: reversible and irreversible inactivation. J. Immunol., 76, 200-208

53. Hodyra-Stefaniak, K., Miernikiewicz, P., Drapała, J., Drab, M., Jończyk-Matysiak, E., Lecion, D., Kaźmierczak, Z., Beta, W., Majewska, J., Harhala, M., et al. (2015) Mammalian Host-VersusPhage immune response determines phage fate in vivo. Sci. Rep., 5, 14802

54. Sokoloff, A. V., Zhang, G., Sebestyén, M. G. and Wolff, J. A. (2000) The interactions of peptides with the innate immune system studied with use of T7 phage peptide display. Mol. Ther., 2, 131-139

55. Merril, C. R., Biswas, B., Carlton, R., Jensen, N. C., Creed, G. J., Zullo, S. and Adhya, S. (1996) Long-circulating bacteriophage as antibacterial agents. Proc. Natl. Acad. Sci. USA, 93, 3188-3192

56. Vitiello, C. L., Merril, C. R. and Adhya, S. (2005) An amino acid substitution in a capsid protein enhances phage survival in mouse circulatory system more than a 1000-fold. Virus Res., 114, 101-103

57. Capparelli, R., Ventimiglia, I., Roperto, S., Fenizia, D. and Iannelli, D. (2006) Selection of an Escherichia coli O157:H7 bacteriophage for persistence in the circulatory system of mice infected experimentally. Clin. Microbiol. Infect., 12, 248-253

58. Capparelli, R., Parlato, M., Borriello, G., Salvatore, P. and Iannelli, D. (2007) Experimental phage therapy against Staphylococcus aureus in mice. Antimicrob. Agents Chemother., 51, 2765-2773

59. Łusiak-Szelachowska, M., Żaczek, M., Weber-Dąbrowska, B., Międzybrodzki, R., Kłak, M., Fortuna, W., Letkiewicz, S., Rogóż, P., Szufnarowski, K., Jończyk-Matysiak, E., et al. (2014) Phage neutralization by sera of patients receiving phage therapy. Viral Immunol., 27, 295-304

60. Loc-Carrillo, C. and Abedon, S. T. (2011) Pros and cons of phage therapy. Bacteriophage, 1, 111-114

61. Hagens, S. and Bläsi, U. (2003) Genetically modified filamentous phage as bactericidal agents: a pilot study. Lett. Appl. Microbiol., 37, $318-323$

62. Hagens, S., Habel, A., von Ahsen, U., von Gabain, A. and Bläsi, U. (2004) Therapy of experimental pseudomonas infections with a nonreplicating genetically modified phage. Antimicrob. Agents Chemother., 48, 3817-3822

63. Matsuda, T., Freeman, T. A., Hilbert, D. W., Duff, M., Fuortes, M., Stapleton, P. P. and Daly, J. M. (2005) Lysis-deficient bacteriophage therapy decreases endotoxin and inflammatory mediator release and improves survival in a murine peritonitis model. Surgery, 137, 639-646

64. Westwater, C., Kasman, L. M., Schofield, D. A., Werner, P. A., Dolan, J. W., Schmidt, M. G. and Norris, J. S. (2003) Use of genetically engineered phage to deliver antimicrobial agents to bacteria: an alternative therapy for treatment of bacterial infections. Antimicrob. Agents Chemother., 47, 1301-1307
65. Moradpour, Z., Sepehrizadeh, Z., Rahbarizadeh, F., Ghasemian, A., Yazdi, M. T. and Shahverdi, A. R. (2009) Genetically engineered phage harbouring the lethal catabolite gene activator protein gene with an inducer-independent promoter for biocontrol of Escherichia coli. FEMS Microbiol. Lett., 296, 67-71

66. Kasman, L. M., Kasman, A., Westwater, C., Dolan, J., Schmidt, M. G. and Norris, J. S. (2002) Overcoming the phage replication threshold: a mathematical model with implications for phage therapy. J. Virol., 76 , $5557-5564$

67. Krom, R. J., Bhargava, P., Lobritz, M. A. and Collins, J. J. (2015) Engineered phagemids for nonlytic, targeted antibacterial therapies. Nano Lett., 15, 4808-4813

68. Tamma, P. D., Cosgrove, S. E. and Maragakis, L. L. (2012) Combination therapy for treatment of infections with gram-negative bacteria. Clin. Microbiol. Rev., 25, 450-470

69. Gaj, T., Gersbach, C. A. and Barbas, C. F. III (2013) ZFN, TALEN, and CRISPR/Cas-based methods for genome engineering. Trends Biotechnol., 31, 397-405

70. Citorik, R. J., Mimee, M. and Lu, T. K. (2014) Sequence-specific antimicrobials using efficiently delivered RNA-guided nucleases. Nat. Biotechnol., 32, 1141-1145

71. Bikard, D., Euler, C., Jiang, W., Nussenzweig, P. M., Goldberg, G. W., Duportet, X., Fischetti, V. A. and Marraffini,L. A. (2014) Development of sequence-specific antimicrobials based on programmable CRISPRCas nucleases. Nat. Biotechnol., 32, 1146-1150

72. Yosef, I., Manor, M., Kiro, R. and Qimron, U. (2015) Temperate and lytic bacteriophages programmed to sensitize and kill antibioticresistant bacteria. Proc. Natl. Acad. Sci. USA, 112, 7267-7272

73. Yacoby, I., Bar, H. and Benhar, I. (2007) Targeted drug-carrying bacteriophages as antibacterial nanomedicines. Antimicrob. Agents Chemother., 51, 2156-2163

74. Lu, T. K. and Collins, J. J. (2009) Engineered bacteriophage targeting gene networks as adjuvants for antibiotic therapy. Proc. Natl. Acad. Sci. USA, 106, 4629-4634

75. Edgar, R., Friedman, N., Molshanski-Mor, S. and Qimron, U. (2012) Reversing bacterial resistance to antibiotics by phage-mediated delivery of dominant sensitive genes. Appl. Environ. Microbiol., 78, 744 751

76. Libis, V. K., Bernheim, A. G., Basier, C., Jaramillo-Riveri, S., Deyell, M., Aghoghogbe, I., Atanaskovic, I., Bencherif, A. C., Benony, M., Koutsoubelis, N., et al. (2014) Silencing of antibiotic resistance in $E$. coli with engineered phage bearing small regulatory RNAs. ACS Synth. Biol., 3, 1003-1006

77. Bárdy, P., Pantůček, R., Benešík, M. and Doškař, J. (2016) Genetically modified bacteriophages in applied microbiology. J. Appl. Microbiol., $121,618-633$

78. Frey, J. (2007) Biological safety concepts of genetically modified live bacterial vaccines. Vaccine, 25, 5598-5605

79. http://www.fda.gov/BiologicsBloodVaccines/Vaccines/ApprovedProducts/ucm293952.htm

80. http://www.ema.europa.eu/docs/en_GB/document_library/EPAR_Public assessment report/human/002617/WC500158413.pdf 